\title{
The potential distribution of the Vulnerable African lion Panthera leo in the face of changing global climate
}

\author{
A. Townsend Peterson, Thomas Radocy, Erin Hall \\ Julian C. Kerbis Peterhans and Gastone G. Celesia
}

\begin{abstract}
The objective of this study is to estimate possible impacts of global climate change on the geographical distribution of the African lion Panthera leo in the coming decades. Current lion population occurrence data across Africa and distributions of lions in historical times (6,000-100 years before present) were obtained from the literature and integrated with data on present-day climates to generate ecological niche models. Models based on distributions of African lions were tested for predictive ability based on various subsetting approaches and were projected across Asia, Africa and Europe, to retrodict the distribution of the species for the past 6,000 years. These models were highly accurate, giving confidence in future projections. Future potential distributions were predicted by projecting ecological niche models onto three climate scenarios of future greenhouse gas emissions based on eight climate models for the years 2040-2070. The prediction was of relative range stability into the future: few new areas were identified as becoming suitable for the species but large areas of southern Africa and West Africa are expected to become less suitable. Predictions of effects of climate change on potential distributions of lions may assist conservation efforts by clarifying options for mitigation and response.
\end{abstract}

Keywords Africa, climate change, ecological niche, geographical distribution, lion, Panthera leo

This paper contains supplementary material that can be found online at http://journals.cambridge.org

\section{Introduction}

T ions Panthera leo were widely distributed across Africa, 1 South-west Asia and southern Europe during historical

A. Townsend Peterson (Corresponding author), Thomas RAdocy and ERIN Hall Biodiversity Institute and Department of Ecology and Evolutionary Biology, University of Kansas, Lawrence, Kansas 66045, USA.

E-mail town@ku.edu

Julian C. Kerbis Peterhans* College of Professional Studies, Roosevelt University, Chicago, Illinois, USA

Gastone Celesia Department of Zoology, Field Museum of Natural History, Chicago, Illinois, USA

${ }^{*}$ Also at: Science and Education, Field Museum of Natural History, Chicago, Illinois, USA

Received 19 December 2011. Revision requested 26 April 2012.

Accepted 11 June 2012. First published online 3 June 2014. times. In Africa lions occupied most of the continent except for the central Sahara and the rainforest areas of central Africa (Ray et al., 2005). Sculptures depict Pharaohs killing male lions on the shores of the Nile. Systematic capture and culling of North African lions expanded during the time of the Roman Empire and continued into the 2oth century (Maas, 2010), to the point that they are now extirpated from the region. Human encroachment in the form of population expansion, agricultural activities and urban development has further impinged on the species' habitat and demography in recent decades.

Numbers of African lions are currently estimated to be between 23,000 (Bauer \& Van Der Merwe, 2004) and 39,373 (Chardonnet, 2002) and the species is categorized as Vulnerable on the IUCN Red List (Bauer et al., 2012). At present lion populations are mostly restricted to protected areas, where populations have been essentially stable over the last 3 decades (Ray et al., 2005). Although the species may largely be protected from direct conflict with humans, the potential effects of global climate change on this limited population have not previously been assessed.

Ecological niche modelling offers novel insights into species' potential geographical distributions, extending from the local population perspective to a cross-distributional, continental view (Guisan \& Zimmermann, 2000). Ecological niche models integrate occurrence data from across the range of a species with environmental data, to estimate the conditions under which the species is able to maintain populations without immigration (Soberón \& Peterson, 2005). Whereas distributions of species are the result of the dynamics of many individual populations, only a view taken across the entirety of a species' geographical range can discern the factors that affect its global distribution. As a consequence, ecological niche models offer a perspective on the future conservation status of species.

Here we evaluate the magnitude of potential changes in the distribution of lion populations over the next 4 decades in light of changing climates. We integrate known lion occurrences (Celesia et al., 2009) with present-day climate data to produce estimates of the lion's ecological niche, which we test via independent data sets, including predictions across both space and time. These niche models are then integrated with numerous future climate projections from general circulation models (GCMs) developed 
under the coordination of the Intergovernmental Panel on Climate Change (Solomon et al., 2007). We thus develop a novel, synthetic view of the potential future distribution of lions that takes into account uncertainty and climate model variations. The ability to predict impacts of climate change on potential geographical distributions of lions across regions will provide information useful in intervention and mitigation strategies to enhance their long-term survival.

\section{Methods}

Input data

Occurrence data for this study were drawn from a previous compilation (Celesia et al., 2009) that identified 27 sites across Africa where lion populations have been documented. These sites generally refer to local regions (such as protected areas) where lion populations have been studied in detail, and are divided into low- and high-abundance sites. We also summarized the historical range of the species across Africa, Asia and southern Europe (Yamaguchi et al., 2004). We accumulated occurrence data for lions in historical times (i.e. from 6,000 years before present to c.10o years ago) through a detailed literature search covering scientific journals and books (Supplementary Material 1) and continuing until discovery of new sources and new occurrence localities reached an asymptote. We added geographical coordinates to these occurrences based on consultation of gazetteer data in Google Earth (2014). The point-based geographical references were supplemented with a measure of the uncertainty in the georeference in the form of the radius of a circle including the entire footprint of the locality description (Wieczorek et al., 2004). For example, a site described as 'southern Iberian Peninsula' is best described by the geographical point $39.093^{\circ} \mathrm{N} 3.777^{\circ} \mathrm{W}$ but sites in the southern Iberian Peninsula extend up to $680 \mathrm{~km}$ from this point, and we therefore use this radius as the uncertainty measure. In total 49 separate historical sites were identified and georeferenced at different levels of precision across North Africa, southern Europe, and southern and South-west Asia. We note that although the historical data are attractive as a basis for ecological niche model calibration, the large uncertainty associated with most of the historical records makes such a calibration untenable.

Data describing present-day climatic variation across Africa, Europe and Asia were drawn from the WorldClim climate data archive (Hijmans et al., 2005). This data set is derived from interpolation among weather station data from 14,000-48,00o localities, and provides monthly averages of precipitation and minimum and maximum temperatures for 1960-1990 at three spatial resolutions; we chose the intermediate spatial resolution of $2.5^{\prime}$ for all analyses, to match the approximate precision of the occurrence data. To provide a richer and more directly informative climatic data set for analysis we used the bioclimatic data layers in the WorldClim archive, specifically the summaries of mean annual temperature, mean diurnal temperature range, maximum temperature of warmest month, minimum temperature of coldest month, annual precipitation, and precipitation of wettest and driest months, which we have found to be relatively uncorrelated and information-rich (Jiménez-Valverde et al., 2009). We clipped the environmental data to Sub-Saharan Africa, to delimit the area within which models were calibrated to the approximate area that has been accessible to the species for colonization (Barve et al., 2011).

We projected present-day models onto climate output of GCMs summarizing model projections for anticipated future conditions. The most relevant time period for which model results are available is $2040-2070$. We used futureclimate data products from the World Climate Research Program downscaled (Conservation International, 2014) and provided to us by Y. Nakazawa (pers. comm.). We used three 21st century standard emissions scenarios explored by the Intergovernmental Panel on Climate Change (Houghton et al., 2001), as follows:

Scenario $B_{1} \mathrm{~B}_{1}$ emissions correspond to a convergent world with a global population peaking in mid century and declining thereafter. Concurrent changes in economic structures move towards a service and information economy, reductions in material intensity, and introduction of clean and resource-efficient technologies. The emphasis under this scenario is on global solutions to economic, social and environmental sustainability, including improved equity, but without additional climate initiatives. The B1 scenario assumes the most ecologically friendly future.

Scenario $A_{1} B$ A1 emissions correspond to a future world of very rapid economic growth, global population peaking in mid century and declining thereafter, and rapid introduction of new and efficient technologies. Major underlying themes are convergence among regions, capacity building, and increased cultural and social interactions, with substantial reduction in regional differences in per capita income. The $\mathrm{AlB}$ scenario emphasizes balance across all energy sources.

Scenario $\mathrm{A}_{2} \mathrm{~A}_{2}$ emissions correspond to a scenario of a very heterogeneous world, characterized by self-reliance and preservation of local identities. Because fertility patterns across regions converge slowly, global populations are continuously increasing; economic development is regionally oriented, and per capita economic growth and technological change are more fragmented and slower than under the other scenarios. The $\mathrm{A}_{2}$ scenario is 
characterized by a future world still heavily dependent on fossil fuel consumption, and essentially maintains the current status quo.

These scenarios of emissions form the basis for model projections of future changes in climate-determined potential distributions of species, via future climate projections from GCM outputs. We thus explore these three scenarios of differing magnitudes of possible climate change in tandem with eight GCMs, each effectively a distinct simulation of global climate processes, to be able to consider effects of model variation in influencing future climate estimates. We projected present-day ecological niche estimates onto these 3 scenarios $\times 8 \mathrm{GCMs}$, although four of the emissions scenario $\times$ climate model combinations were unavailable (Table 1).

\section{Ecological niche modelling}

We used the Genetic Algorithm for Rule-Set Prediction (GARP) for generating ecological niche models (Stockwell \& Peters, 1999). Although GARP has been criticized in comparative analyses (Elith et al., 2006), more careful comparisons taking into account different sorts of prediction error have indicated that the differences in performance are largely artefactual (Peterson et al., 2008). Furthermore, for the present application, GARP's ability to project niche model results onto 10 future climate surfaces greatly improves efficiency of the future-projection process compared with other algorithms (Phillips et al., 2006). As a consequence, GARP was an ideal choice as a niche modelling platform for this study.

GARP is an evolutionary computing method that builds ecological niche models based on non-random associations between known occurrence points for species and sets of digital raster coverages describing ecological landscapes. Occurrence data are used by GARP as follows: $50 \%$ of occurrence data points are set aside for evaluation of quality of replicate models for development of consensus models, and 50\% are used for developing models. 'Pseudoabsence' points (i.e. pixels selected from the set of areas lacking known presences) are also sampled. Presence and absence points are divided into equal portions for rule generation and model testing (Stockwell \& Peters, 1999).

Initial rules are created by applying methods chosen randomly from a set of inferential tools (e.g. logistic regression, bioclimatic rules). The genetic algorithm consists of specially defined operators (e.g. crossover, mutation) that modify the initial rules; the resulting models have therefore 'evolved'. After each modification, the quality of the rule is tested (maximizing both significance and predictive accuracy) and a size-limited set of best rules is retained. Because rules are tested based on independent data (intrinsic test data), performance values reflect the expected performance of the rule, an independent verification that gives a more reliable estimate of true rule performance. The final result is a set of rules that can be projected onto a map to estimate the potential geographical distribution of the species under investigation, which must be distinguished carefully from estimates of the actual distribution of the species, which would take into account considerations of accessibility and barriers to dispersal (Soberón \& Peterson, 2005).

Following best-practice recommendations for appropriate consensus of replicate models built from different subsamples of occurrence data (Anderson et al., 2003), we developed 100 replicate random-walk GARP models, and filtered out $90 \%$ of the replicate models based on consideration of error statistics, as follows. The initial filter removes models that omit (omission error $=$ predicting absence in areas of known presence) over much as measured with extrinsic testing data, and a second filter based on an index of commission error (= predicting presence in areas of known absence), in which models predicting very large or very small areas are removed from consideration. Specifically, we used a soft omission threshold of $20 \%$, and a $50 \%$ retention based on commission considerations; the result was 10 'best subsets' binary model predictions that

TABLE 1 Summary of trends in temperature and precipitation anticipated from general circulation models and Special Report on Emissions Scenarios explored in the future-climate portion of this study. To illustrate the likely effects anticipated under each scenario we present mean change in temperature/precipitation based on values manifested at 244 random points across Africa. A blank cell indicates that model output was unavailable for analysis.

\begin{tabular}{|c|c|c|c|}
\hline General circulation model & $\begin{array}{l}\text { A2 scenario } \\
\left({ }^{\circ} \mathrm{C} / \mathrm{mm}\right)\end{array}$ & $\begin{array}{l}\text { A1B scenario } \\
\left({ }^{\circ} \mathrm{C} / \mathrm{mm}\right)\end{array}$ & $\begin{array}{l}\text { B1 scenario } \\
\left({ }^{\circ} \mathrm{C} / \mathrm{mm}\right)\end{array}$ \\
\hline Bjerknes Centre for Climate Research, Norway, BCM2.0 Model & $1.6 / 9.0$ & $1.7 / 0.0$ & $1.3 / 15.0$ \\
\hline CSIRO Atmospheric Research, Australia, Mk3.0 Model & $1.7 /-8.5$ & $1.5 / 0.0$ & $1.2 /-6.0$ \\
\hline CSIRO Atmospheric Research, Australia, Mk3.5 Model & $2.5 /-12.5$ & $2.5 /-9.5$ & $2.1 /-1.5$ \\
\hline Institute for Numerical Mathematics, Russia, INMCM3.0 Model & $2.2 / 0.0$ & $2.2 /-2.0$ & $1.7 / 1.0$ \\
\hline NASA Goddard Institute for Space Studies, ModelE20/Russell & & $1.8 /-3.0$ & $1.4 / 0.0$ \\
\hline CCSR/NIES/FRCGC, Japan, MIROC3.2, high resolution & & $2.8 / 5.5$ & $2.4 / 8.0$ \\
\hline CCSR/NIES/FRCGC, Japan, MIROC3.2, medium resolution & & $1.8 / 13$ & $1.4 / 17.0$ \\
\hline National Center for Atmospheric Research, CCSM3.0 & & $2.7 / 61$ & $1.9 / 42.5$ \\
\hline
\end{tabular}




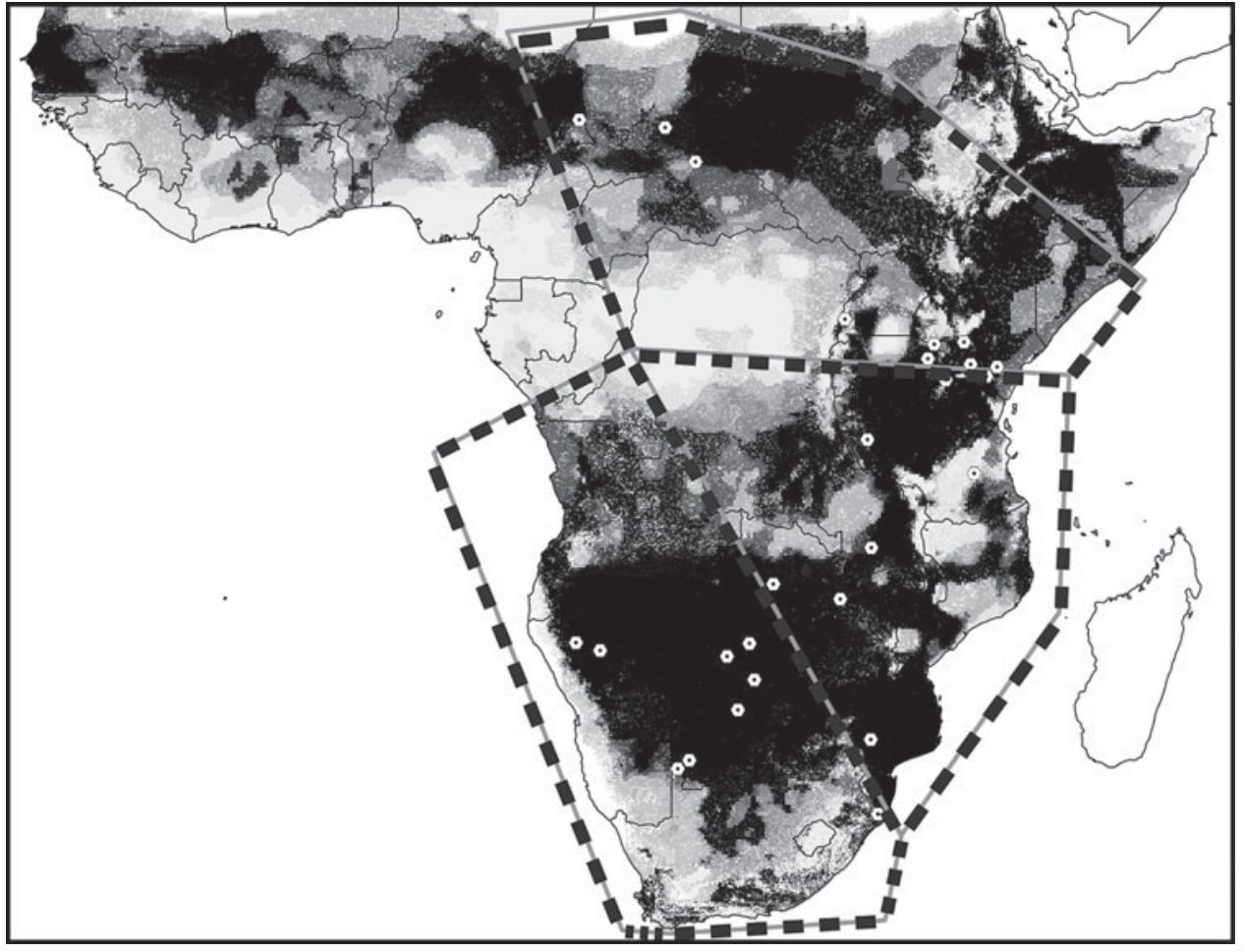

FIG. 1 Summary of spatial splits used to subset data points across the range of the lion Panthera leo across Africa: black-and-grey lines delimit the north, central, and south sectors of the species' range. Dotted white circles represent the occurrence data available. The shading is an example prediction (spatial split omitting the central sector), in which white indicates prediction of lack of suitable conditions, black indicates full model agreement in predicting suitability of conditions, and shades of grey indicate intermediate levels of model agreement. were summed to produce a best estimate of geographical prediction.

\section{Testing model predictions}

Because ecological niche models must be tested for predictive ability prior to interpretation and exploration (Peterson, 2005), particularly for transferability challenges such as this one, we tested the ability of our present-day African models to anticipate distribution of lions across regions from which occurrence data were withheld from model calibration. We used three such manipulations: (1) random subsetting of available occurrence points into training and testing sets, (2) spatial stratification of known occurrences into training and testing areas, and (3) ability to predict the full spatial footprint of the historical distribution of lions across Africa, Asia and Europe. Details of these tests are as follows.

We tested the internal consistency of lion ecological niche characteristics by means of $k$-fold subsetting in two ways. The first was by division into two equal portions at random to test the ability of models to anticipate the spatial distribution of the species, were we to have double the density of occurrence data; this random subsetting was repeated five times. Secondly, we separated available occurrence information spatially into three arbitrary regions of equal sample size: occurrence information from each sector was set aside, and models developed based on the occurrences in the other two sectors. The occurrence information from the reserved sector was then overlaid on the predictions within the range of that sector to test the predictive ability of the model. Given the crescent-shaped nature of the distribution of lions in Africa, for these spatially stratified tests of predictive ability across unsampled areas, we separated known occurrences into three groups of equal sample size ( $\mathrm{n}=9$ points each) by dividing the continent into radial sectors surrounding a point in the Congo Basin, leaving equal numbers of points in each sector (Fig. 1). This arbitrary spatial subdivision into three sectors permits us to test ability of models to predict across different sectors of the species' range.

Because our models are based only on data documenting presence of lions across Africa, and because information documenting absence of climatic suitability for lion populations across Africa are unavailable, customary approaches to model validation (e.g. receiver operating characteristic, kappa statistics) are neither appropriate nor applicable (Lobo et al., 2008). As a consequence, we modified the receiver operating characteristic (ROC) approach so as not to depend on absence data by recasting the (1-specificity) axis as the proportional area predicted as suitable following Phillips et al. (2006) and Peterson et al. (2008). The area under the curve (AUC) of traditional ROC approaches undervalues models that do not provide predictions across the entire spectrum of proportional areas in the study area (Peterson et al., 2008). In addition, traditional ROC approaches incorrectly weight the two error components (omission and commission) equally (Peterson et al., 2008). As a consequence, we used the partial-area ROC approach 
TABLE 2 Summary of results of subset-based tests of predictive ability of models. Random splits divided the available occurrence data into two equal subsets but without spatial separation; this testing process was repeated five times based on different random splits (testing $\mathrm{n}=13$ ). Spatial splits divided the available occurrence data into three subsets $(\mathrm{N}, \mathrm{C}$ and $\mathrm{S}$ sectors), each holding nine points (Fig. 1). The proportion of 1,00o bootstrap replicates with AUC ratio $<1$ was used as an estimate of P. AUC indicates the area under the receiver operating characteristic plot (see text for further details).

\begin{tabular}{llr}
\hline Specific test & Minimum AUC ratio & \multicolumn{1}{c}{$\mathrm{P}$} \\
\hline Random split 1 & 0.976 & 0.064 \\
Random split 2 & 1.141 & $<0.001$ \\
Random split 3 & 1.092 & $<0.001$ \\
Random split 4 & 0.960 & 0.640 \\
Random split 5 & 1.243 & $<0.001$ \\
C and S predict N & 1.335 & $<0.001$ \\
S and N predict C & 0.953 & 0.377 \\
N and C predict S & 0.984 & 0.402 \\
\hline
\end{tabular}

that evaluates only over the range of the prediction and potentially allows differential weighting of the two error components (Peterson et al., 2008). A more detailed treatment of model evaluation considerations and preferred techniques is provided in Peterson et al. (2011).

We performed partial ROC analyses for each of the five random-subsetting model predictions and the three spatially-stratified predictions, based on the independent sets of testing points not used to train the models. Partial ROC analyses (Peterson et al., 2008) were developed principally to remove the focus of more traditional ROC analyses (Fielding \& Bell, 1997), which include what are generally considered irrelevant predictions (i.e. massive underprediction with large omission errors) that can bias conclusions, although other concerns certainly remain (Lobo et al., 2008). We calculated these values using software developed by N. Barve (pers. comm.; available upon request from the authors); we present our results as the ratio of the model AUC to the null expectation (AUC ratio; Peterson et al., 2008). AUC ratios were limited to the proportional area over which models made predictions, and were based on expected error parameters of $\mathrm{E}=5 \%$ (Peterson et al., 2008). Bootstrapping to evaluate the statistical significance of AUC ratios compared to the null model was performed by resampling $50 \%$ of the test points, with replacement, 1,00o times from the overall pool of testing data; one-tailed probabilities associated with AUC ratios were assessed by counting numbers of bootstrap replicates with AUC ratios $<_{1}$ (hereafter referred to as $\mathrm{P}$, Table 2).

We tested for prediction of the full distribution of lions based on the historical occurrence data described above. We used all available present-day occurrence data (Celesia et al., 2009) to train models, and projected those models more broadly across North Africa, Europe, and southern and South-west Asia. The goal was to evaluate the degree to which historical distributions of lions could be anticipated from current distributions. As explained in the input data section above, we restricted these analyses to the past 6,00o years, a period over which global climates have been relatively stable. These predictions, which depended rather critically on the uncertainty estimates surrounding the often-imprecise historical locality descriptors (e.g. 'Balkans', 'North Africa', 'southern Iberian Peninsula'), were evaluated only visually and not via specific statistical tests; however, we note that a positive result on these tests (i.e. that models based on the current distribution of the species can anticipate the entire historical distributional area) would be taken as indicating that no major biases are introduced by our use of current distribution data instead of the broader historical distribution of the species.

\section{Future projections}

After niche models were calibrated and tested as described above, we explored their implications for future potential distribution of lions. We used all available occurrence data (Celesia et al., 2009) to train present-day models, and projected each model onto all available scenarios and models of future (2050) climate conditions (Table 1). These projections were summarized in terms of future potential distribution areas, as well as in terms of variation among emission scenarios and among models.

To estimate uncertainty in future projections we focused on pixel-by-pixel variation in future projections among alternative models under a particular emissions scenario. We took different climate models (e.g. Hadley, NCAR) as independent efforts to reconstruct the dynamics of a complex system, and inspected the variation between climate models carefully. Specifically, for a given prediction (e.g. 7 of 10 replicate models indicating suitability of a site under a given emissions scenario) we calculated the proportion of available models that disagreed with the modal prediction (in this case, 3 of 10). This simple measure of uncertainty nonetheless summarizes the degree to which individual models may vary from the modal prediction for a given site.

\section{Results}

Initial tests of model predictivity based on subsets of available occurrence data indicated generally significant predictive ability of models (Table 2, Fig. 1). Specifically, with random subsetting, three of the tests showed predictive ability that was highly significantly better than random expectations $(\mathrm{P}<0.001)$, one was marginally significantly better than random $(P=0.064)$, and one was not significant $(\mathrm{P}=0.640)$. Spatial subsetting exercises yielded more mixed 


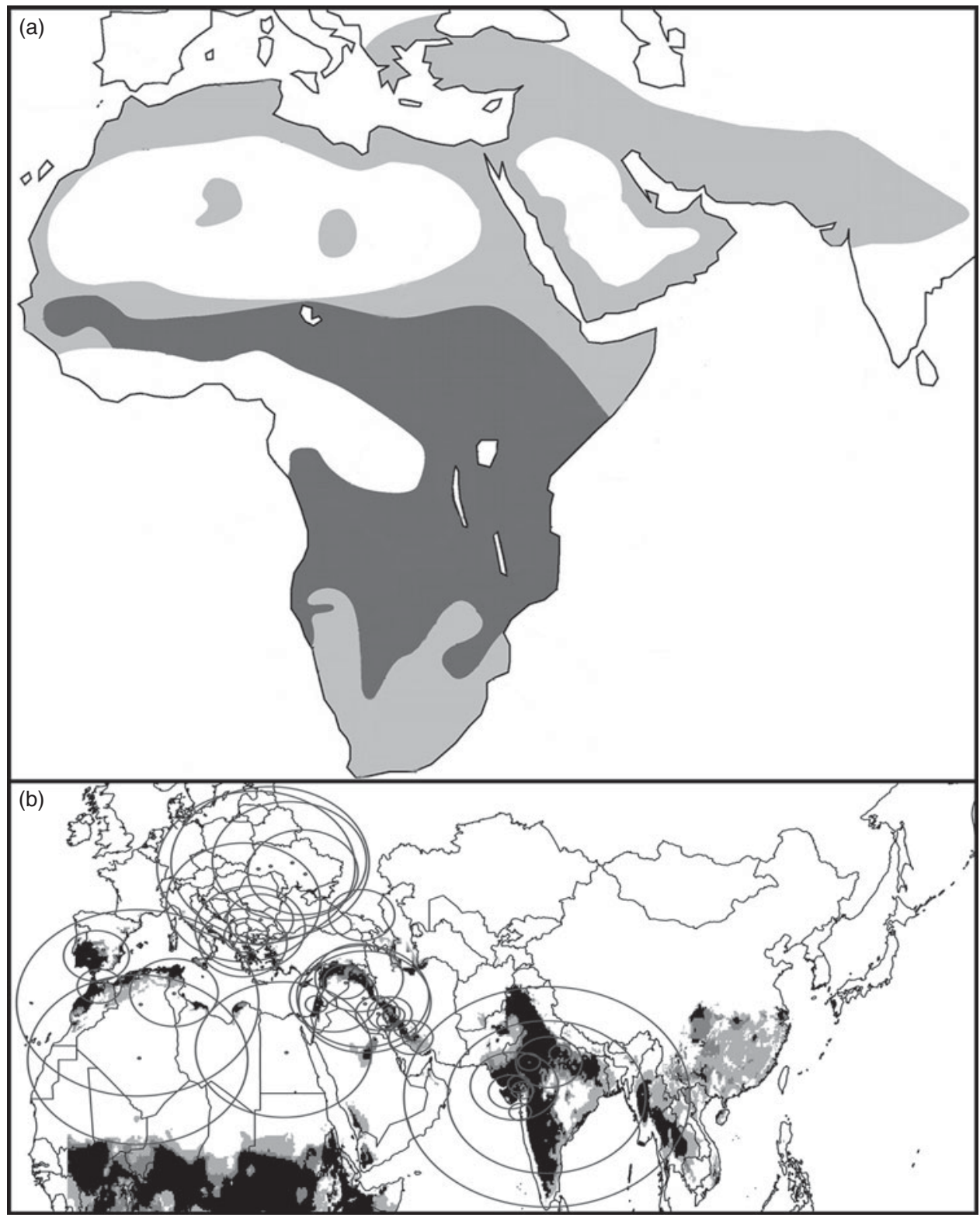

FIG. 2 Spatial test of model predictions, in which occurrences from across the present-day African range of lions were used to train a model that was in turn projected (transferred) across the historical Asian, European and North African range of the species (dark grey, present range; light grey, historical range). (a) Summary of historical range modified from Barnett et al. (2006b) and reproduced here with permission. (b) Niche model results (white $=$ unsuitable, light grey $=$ at least one replicate model predicts suitability, medium grey $=$ most replicate models predict suitability, black $=$ all replicate models predict suitability). Occurrence data (Supplementary Material 1) from the past 6,000 years are shown as points and circles and represent approximate precision of georeferencing (i.e. a smaller circle indicates more precise georeferencing).

results, with one of three tests yielding an AUC ratio significantly elevated above null expectations. We suspect that these predictive failures result from incomplete representation of conditions manifested in the regions over which the models were trained, given strong environmental gradients across eastern and southern Africa.

The final suite of tests was based on all occurrence data available (Celesia et al., 2009). We projected current African distribution models broadly across Asia, Europe and Africa to investigate the degree to which they could recover the broader portions of the historical distribution of the species (Fig. 2). These models were successful in reconstructing potential distribution of lions in North Africa, the Iberian Peninsula, parts of the Balkans, the Middle East, and the Indian subcontinent (Barnett et al., 2006b; Schnitzler, 2011). Although our evaluation of these predictions was only in visual terms, all known historical distributional areas were recovered, except for occurrences in the Balkan region. Whereas predictive failure in the Balkans could be a result of limited environmental representation in the training region (eastern and southern Africa), we suspect that the reliability of the historical data may actually be the cause; i.e. these particular occurrences were based on cave art and artefacts (Yamaguchi et al., 2004; Schnitzler, 2011) and may reflect trade or cultural transmission, rather than actual occurrence of lions. Hence, no major biases appear to be introduced by basing models on current distribution (i.e. including the effects of massive reduction in range caused by humans) instead of the full historical distribution.

We projected present-day models equivalent to those used in the backwards predictions just discussed onto scenarios of future climate conditions (Fig. 3), calculating 


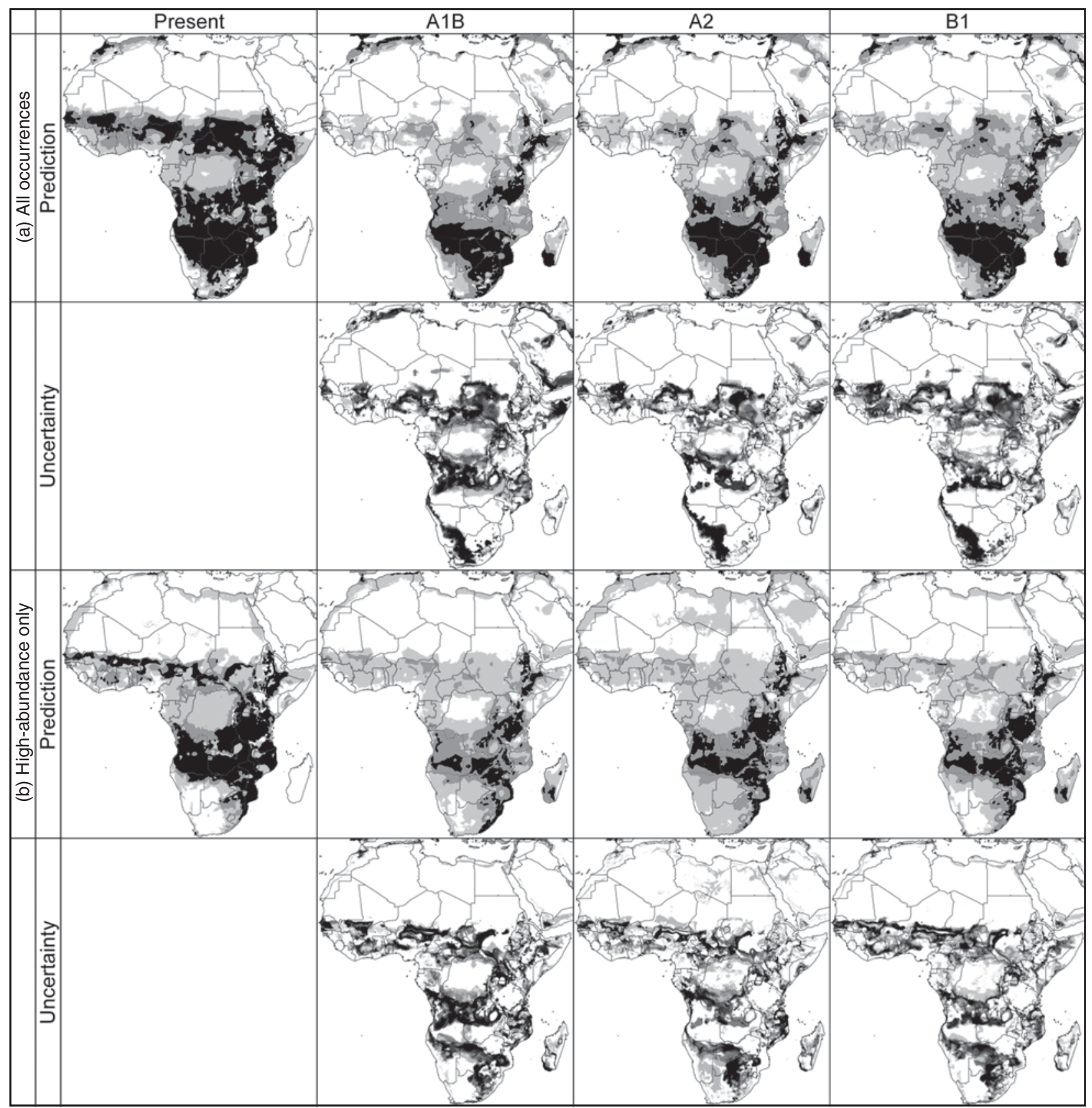

FIG. 3 Summary of present and future projections ( $\mathrm{A}_{1} \mathrm{~B}, \mathrm{~A} 2$, and $\mathrm{B}_{1}$ emissions scenarios; see text for details) of models based on (a) all occurrences of lions, and (b) high-abundance occurrences of lions (white $=$ unsuitable, light grey $=$ at least one replicate model predicts suitability, medium grey $=$ most replicate models predict suitability, black $=$ all replicate models predict suitability) . Accompanying each future projection is an estimate of the uncertainty in those projections, calculated as model disagreement in a given prediction $($ white $=$ high confidence, grey $=$ intermediate values, black $=$ greatest uncertainty).

both the consensus prediction among the eight climate models used to anticipate future climate conditions and the uncertainty of those predictions. The predictions into the future generally indicate relative range stability, in the sense that no broad new areas would become suitable for lions. Many currently suitable areas, however, particularly in West Africa and southern Africa, are expected to decline in suitability (Fig. 3). Uncertainty in these predictions, however, is also highest in these areas, so variation between models means there is less confidence in the predictions for these areas. Models based on all occurrences were similar in broadest terms to those based on high-abundance sites only; however, the high-abundance models identified more restricted areas in West Africa and southern Africa, where the species is known to have lower population densities (Celesia et al., 2009). 


\section{Discussion}

The task of developing models predicting the future potential distribution of a species under changing conditions is not simple. In the first place, the models must be well-trained; i.e. they must include all the dimensions of environmental conditions under which the species can maintain populations (Soberón \& Peterson, 2005). To achieve this representativeness, models must be trained over the broadest environmental spectrum possible, in this case the entire geographical distribution of the species. Testing and validation of this predictive ability is key to bolstering the validity of these model projections. In our analysis the random and spatial subsetting exercises yielded mixed results, which we suspect reflects the fact that our occurrence data set was not rich in numbers of occurrences. This small training data set weakens statistical tests and makes models more vulnerable to incomplete niche characterization.

The most thorough test of this full representation of environments in ecological niche models is that of predictability of responses to distributional and environmental phenomena when challenged with independent testing data sets. In this case, the spatial subsetting exercises showed mixed success, perhaps owing to insufficient representation of environments in the training regions or perhaps simply to small sample sizes. However, in exercises in which occurrences from the entire present-day African range were projected broadly across North Africa, southern and south-eastern Asia and southern Europe, the models were able to anticipate all major features of the broader historical distribution of the species, which inspires confidence in their predictive ability and in the representativeness in ecological dimensions of the niches that were estimated for the species.

In projecting present-day niche models onto anticipated future conditions there is little to inspire optimism regarding the future of lions. A summary of the phenomena anticipated is that the broadest areas of suitable conditions (southern Africa) are projected to become less suitable because of climate change. Moreover, a broad swath of the species' potential distributional area (West Africa) is anticipated to become distinctly less suitable or even uninhabitable.

Looking closer at future-trend projections from our models in what could be considered the heart of the distribution of lions (Kenya and Tanzania; Celesia et al., 2009), we see that climate change effects on distributional potential is projected to be more neutral than the picture across the range of the species (Supplementary Figs. S1-S2). In the key reserves of the Serengeti and Masai Mara, climate change effects projected from our models were close to neutral. Elsewhere, however, our model projections were less neutral, as can be appreciated by a view of southern Africa, where the Etosha Pan, Lake Opnono, Cuvelai Drainage, Kalahari Gemsbok, and Kgalagadi Transfrontier Park areas are expected to decline substantially in suitability for lions (Supplementary Figs. S1-S2). In this sense, multinational investments in conservation of lions are best focused in the East African reserves that are most likely to be able to sustain lion populations in the medium term.

African lion populations are now essentially confined to protected areas, where populations have been relatively stable over the past 3 decades (Ray et al., 2005). The main effort of conservation has been to protect these areas from habitat conversion and human-wildlife conflicts (Mesochina et al., 2010). Our models estimate the effects of climate changes on sustainability of lions in these protected areas. These tools can be used for the management and conservation of ecosystems: if well-supported models predict that a specific set of climate changes would result in a region becoming less suitable, then regional strategic plans can be developed to protect the threatened population, which is particularly relevant in the case of large predators (Hayward et al., 2007).

Our models assessed direct effects of climate change on potential distributions of lions, yet secondary influences also affect their survival, and require attention. Anticipated climate changes in Africa are generally in the direction of increased temperature and decreased rainfall (Table 1). Droughts reduce prey availability, at least for lion populations that focus on migratory prey, resulting in an increase in mortality of cubs and older lions from starvation (Bertram, 1973; Dunham, 1992; Funston \& Mills, 2006). Droughts also affect the survival of livestock, making pastoralists less tolerant of depredation by lions. Kenya's Minister of Forestry and Wildlife was recently quoted by The Daily Nation regarding the effects of winter droughts in 2010:

The communities had lost $80 \%$ of their livestock to the drought; when the lions and hyenas turned to the remaining livestock, the communities were distressed and attacked them in turn... The drought took a heavy toll on both wild animals and the habitats we care for. Besides, it also adversely affected the livestock of communities living adjacent to national parks and reserves.

Thus, and particularly in light of the broader-scale effects documented in this paper, climate change could increase human-lion conflicts. Ogutu et al. (2011) have studied wildlife populations in the Masai Mara National Reserve and adjoining pastoral ranches since 1977; they found that almost all wildlife species have declined to a third or less of their former abundance and that human influences appeared to be the main cause.

In 1994 a canine distemper virus (CDV) epidemic in Serengeti lions resulted in the death of one third of the population, and in 2001 a second high-mortality CDV 
epidemic struck the nearby Ngorongoro Crater lion population (Munston et al., 2008). The outbreak was apparently the result of a convergence of extreme drought conditions, infestation of Babesia (a tick-borne intraerythrocytic protozoan), and CDV infection. Munston et al. (2008) concluded that severe climate changes may 'alter historic host-pathogen relationships and synchronize the temporal and spatial convergence of multiple infectious agents, triggering epidemics with far greater mortality than those from single pathogens'. These examples emphasize the close relationship between climate change, disease and human-animal interactions (Scholte, 2011). The recognition that local-scale phenomena such as disease outbreaks may form part of broader-scale trends can guide efforts to mitigate negative effects of climate change on species such as lions.

\section{Acknowledgements}

The Program for Climate Model Diagnosis and Intercomparison and the World Climate Research Program were instrumental in opening access to climate model outputs, and Conservation International generously invested in downscaling the raw outputs, providing access to this rich source of data. Y. Nakazawa calculated bioclimatic variables from the downscaled data and kindly provided copies to us. This work was supported in part by a grant to ATP from Microsoft Research.

\section{References}

Anderson, R.P., Lew, D. \& Peterson, A.T. (2003) Evaluating predictive models of species' distributions: criteria for selecting optimal models. Ecological Modelling, 162, 211-232.

Barnett, R., Yamaguchi, N., Barnes, I. \& Cooper, A. (2006a) Lost populations and preserving genetic diversity in the lion Panthera leo: implications for its ex situ conservation. Conservation Genetics, 7, 507-514.

Barnett, R., Yamaguchi, N., Barnes, I. \& Cooper, A. (2006b) The origin, current diversity and future conservation of the modern lion (Panthera leo). Proceedings of the Royal Society B, 273 , 2119-2125.

Barnett, R., Yamaguchi, N., Shapiro, B. \& Sabin, R. (2008) Ancient DNA analysis indicates the first English lions originated from North Africa. Contributions to Zoology, 77, 7-16.

Barve, N., Barve, V., Jiménez-Valverde, A., Lira-Noriega, A., Maher, S.P., Peterson, A.T. et al. (2011) The crucial role of the accessible area in ecological niche modelling and species distribution modelling. Ecological Modelling, 222, 1810-1819.

Bauer, H., Nowell, K. \& Packer, C. (2012) Panthera leo. In IUCN Red List of Threatened Species v. 2013.2. Http://www.iucnredlist.org [accessed 14 March 2014].

Bauer, H. \& Van Der Merwe, S. (2004) Inventory of free-ranging lions Panthera leo in Africa. Oryx, 38, 26-31.

Benecke, N. (1999) The Holocene History of the European Vertebrate Fauna: Modern Aspects of Research, Workshop 6-9 April 1998, Berlin, pp. 223-235. Verlag Marie Leidorf GmbH, Rahden, Germany.
Bertram, B. (1973) Lion population regulation. East African Wildlife Journal, 11, 215-225.

Celesia, G., Peterson, A.T., Kerbis-Peterhans, J. \& Gnoske, T. (2009) Climate and landscape correlates of African lion (Panthera leo) demography. African Journal of Ecology, 48, 58-71.

Chardonnet, P. (2002) Conservation of the African lion. International Foundation for the Conservation of Wildlife, Paris, France.

Conservation International (2014) Http://futureclimates. conservation.org [accessed 14 March 2014].

Dunham, K. (1992) Response of a lion (Panthera leo) population to changing prey availability. Journal of Zoology, 227, 330-333.

Elith, J., Graham, C.H., Anderson, R.P., Dudik, M., Ferrier, S., Guisan, A. et al. (2006) Novel methods improve prediction of species' distributions from occurrence data. Ecography, 29, 129-151.

Fielding, A.H. \& BelL, J.F. (1997) A review of methods for the assessment of prediction errors in conservation presence/absence models. Environmental Conservation, 24, 38-49.

Funston, P. \& Mills, M.G.L. (2006) The influence of lion predation on the population dynamics of common large ungulates in the Kruger National Park. South African Journal of Wildlife Research, $36,9-22$.

Google Earth (2014) Http://www.google.com/earth [accessed 14 March 2014].

Guggisberg, C.A.W. (1975) Wild Cats of the World. Taplinger Publishing, New York, USA.

Guisan, A. \& Zimmermann, N.E. (200o) Predictive habitat distribution models in ecology. Ecological Modelling, 135, 147-186.

Hayward, M., O’Brien, J. \& Kerleya, G.I.H. (2007) Carrying capacity of large African predators: predictions and tests. Biological Conservation, 139, 219-229.

Hijmans, R.J., Cameron, S.E., Parra, J.L., Jones, P.G. \& Jarvis, A. (2005) Very high resolution interpolated climate surfaces for global land areas. International Journal of Climatology, 25, 1965-1978.

Houghton, J.T., Ding, Y., Griggs, D.J., Noguer, M., van Der Linden, P.J., DAI, X. et al. (eds) (2001) Climate Change 2001: The Scientific Basis. Cambridge University Press, Cambridge, UK.

Jennison, G.M.A. (1937) Animals for Show and Pleasure in Ancient Rome. Manchester University Press, Manchester, UK.

Jiménez-Valverde, A., Nakazawa, Y., Lira-Noriega, A. \& Peterson, A.T. (2009) Environmental correlation structure and ecological niche model projections. Biodiversity Informatics, 6, $28-35$.

KinneAR, N.B. (1920) The past and present distribution of the lion in South Eastern Asia. Journal of the Bombay Natural Historical Society, 27, 33-39.

Lobo, J.M., Jiménez-Valverde, A. \& Real, R. (2008) AUC: a misleading measure of the performance of predictive distribution models. Global Ecology and Biogeography, 17, 145-151.

Ma As, P. (ed.) (2010) The Sixth Extinction. Http://www.petermaas.nl/ extinct [accessed 21 November 2010].

Mesochina, P., Mbangwa, O., Chardonnet, P., Mosha, R., Mtui, B., Drouet, N. et al. (2010) Conservation Status of the Lion (Panthera leo Linnaeus) in Tanzania. SCI Foundation, Paris, France.

Munston, L., Terio, K.A., Kock, R., Mlengeya, T., Roelke, M.E., Dubovi, E. et al. (2008) Climate extremes promote fatal co-infections during canine distemper epidemics in African lions. PLoS ONE, 3, e2545.

Ogutu, J., Owen-Smith, N., Piepho, H.P. \& Said, M.Y. (2011) Continuing wildlife population declines and range contraction in the Mara region of Kenya during 1977-2009. Journal of Zoology, 285, 99-109. 
Peterson, A.T. (2005) Kansas gap analysis: the importance of validating distributional models before using them. Southwestern Naturalist, 50, 230-236.

Peterson, A.T., Papeş, M. \& Soberón, J. (2008) Rethinking receiver operating characteristic analysis applications in ecological niche modelling. Ecological Modelling, 213, 63-72.

Peterson, A.T., Soberón, J., Pearson, R.G., Anderson, R.P., Martínez-Meyer, E., Nakamura, M. \& Araújo, M.B. (2011) Ecological Niches and Geographic Distributions. Princeton University Press, Princeton, USA.

Ray, J., Hunter, L. \& Zigouris, J. (2005) Setting Conservation and Research Priorities for Larger African Carnivores. Wildlife Conservation Society, New York, USA.

Schnitzler, A.E. (2011) Past and present distribution of the North African-Asian lion subgroup: a review. Mammal Review, 41, 220-243.

Scholte, P. (2011) Towards understanding large mammal population declines in Africa's protected areas: a west-central African perspective. Tropical Conservation Science, 4, 1-11.

Soberón, J. \& Peterson, A.T. (2005) Interpretation of models of fundamental ecological niches and species' distributional areas. Biodiversity Informatics, 2, 1-10.

Solomon, S., Qin, D., Manning, M., Chen, Z., Marquis, M., Averyt, K.B. et al. (eds) (2007) Contribution of Working Group I to the Fourth Assessment Report of the Intergovernmental Panel on Climate Change. Cambridge University Press, Cambridge, UK.
Sommer, R.S. \& Benecke, N. (2006) Late Pleistocene and Holocene development of the felid fauna (Felidae) of Europe: a review. Journal of Zoology, 269, 7-19.

Stockwell, D.R.B. \& Peters, D.P. (1999) The GARP modelling system: problems and solutions to automated spatial prediction. International Journal of Geographical Information Science, 13, 143-158.

Sunquist, M. \& Sunquist, F. (2002) Wild Cats of the World. University of Chicago Press. Chicago, USA.

Thomas, N.R. (2004) The Early Mycenaean lion up to date. Hesperia Supplements, 33, 161-206.

Wieczorek, J., Guo, Q. \& Hijmans, R. (2004) The point-radius method for georeferencing locality descriptions and calculating associated uncertainty. International Journal of Geographical Information Science, 18, 745-767.

Yamaguchi, N., Cooper, A., Werdelin, L. \& Macdonald, D.W. (2004) Evolution of the mane and group-living in the lion (Panthera leo): a review. Journal of Zoology, 263, 329-342.

\section{Biographical sketches}

A. Townsend Peterson is a Professor and Curator at the University of Kansas, where Thomas RADOCY and ERIN HALL were undergraduate students. Julian C. Kerbis Peterhans focuses his research on African mammals. Gastone G. Celesia is a retired Professor of Neurology at Loyola University of Chicago, working with aspects of lion ecology. 